\title{
Modeling and Simulation of an induction machine in the abc-reference frame using inversion of a matrix by partitioning
}

\author{
R. Dimitrovski ${ }^{1}$ and M. Luther ${ }^{1}$ \\ ${ }^{1}$ Chair of Electrical Energy Systems \\ University of Erlangen-Nuremberg \\ Konrad-Zuse-Straße 3-5, 91052 Erlangen (Germany) \\ E-mail: robert.dimitrovski@fau.de
}

\begin{abstract}
This paper describes an alternative mathematical model for simulation of a symmetrical induction machine in the $a b c$-reference frame. It has been mathematically proven that the inverted matrix can be computed without the need of matrix inversion. Instead the elements in main diagonal of the inverted matrix are precomputed and the elements of the secondary matrix are calculated only as a function of the rotor angle. The performance of the model has been verified by comparison with the conventional model which uses matrix inversion. The comparison has been done by observing the induction machine variables during free (no-load) acceleration and showed no differences in the results between the two models. The comparison has also shown that model proposed in this paper needs less computational effort.
\end{abstract}

\section{Key words}

Block matrix inversion, $a b c$-reference frame, induction machine, partitioning.

\section{Introduction}

In an electrical machine the stator and rotor winding are mutually coupled and when the rotor changes its position the mutual induction also changes i.e. it is dependent on the rotor position. This fact made the analysis of the electrical machines more difficult. In order to overcome this problem R.H. Park has introduced a new approach for the analysis of electric machines, which eliminates all rotor position-dependent inductances [1]. This approach is known in the literature as the Park's or the $d q 0$ transformation. Due to its advantages, the $d q 0$ transformation, or the $d q 0$ model of an electrical machine, is the favorable choice when it comes to simulation of electric machines.

There are, however, cases where the modeling of the electrical machine in the $a b c$-reference frame is more advantageous even though it requires more computational effort. Such case is, for example, the modeling and analysis of space harmonics. It has been shown in [2] that the $a b c$-reference frame model is in some cases the only possible way for simulating certain space harmonics.

The purpose of this paper is to describe the solving of the machine equations using matrix partitioning and to provide a faster way for the simulation of symmetrical induction machines in the $a b c$-reference frame. This is achieved by eliminating the need for inversion of the inductance matrix. Instead, a mathematical model is developed which pre-calculates the elements of the main diagonal of the inverted matrix and the elements of the secondary diagonal are merely a function of the rotor angle $\theta_{r}$.

The developed model will be verified by comparison with the conventional method which uses matrix inversion.

\section{Mathematical model of the induction machine in abc-reference frame}

The general model of the induction machine is given by the following differential equations for the stator and rotor voltages [3]:

$$
\underbrace{\left[\begin{array}{c}
\mathbf{v}_{\text {abcs }} \\
\mathbf{v}_{\text {abcr }}^{\prime}
\end{array}\right]}_{\mathbf{V}}=\underbrace{\left[\begin{array}{cc}
\mathbf{r}_{\mathrm{s}} & \mathbf{0} \\
\mathbf{0} & \mathbf{r}_{\mathbf{r}}^{\prime}
\end{array}\right]}_{\mathbf{r}} \underbrace{\left[\begin{array}{c}
\mathbf{i}_{\text {abcs }} \\
\mathbf{i}_{\text {abcr }}
\end{array}\right]}_{\mathbf{i}}+\frac{\mathbf{d}}{\mathbf{d t}} \underbrace{\left[\begin{array}{c}
\lambda_{\text {abcs }} \\
\lambda_{\text {abcr }}^{\prime}
\end{array}\right]}_{\lambda}
$$

The matrices of the stator and rotor resistances are defined as:

$$
\mathbf{r}_{\mathbf{s}}=\left[\begin{array}{ccc}
r_{a s} & 0 & 0 \\
0 & r_{b s} & 0 \\
0 & 0 & r_{c s}
\end{array}\right] \mathbf{r}_{\mathbf{r}}^{\prime}=\left[\begin{array}{ccc}
r_{a r}^{\prime} & 0 & 0 \\
0 & r_{b r}^{\prime} & 0 \\
0 & 0 & r_{c r}^{\prime}
\end{array}\right]
$$

The flux linkages for the stator and rotor winding can be expressed in the following matrix form:

$$
\left[\begin{array}{l}
\lambda_{\text {abcs }} \\
\lambda_{\text {abcr }}^{\prime}
\end{array}\right]=\left[\begin{array}{cc}
\mathbf{L}_{\text {ss }} & \mathbf{L}_{\text {sr }}^{\prime}\left(\boldsymbol{\theta}_{\mathbf{r}}\right) \\
\mathbf{L}_{\mathrm{rs}}^{\prime}\left(\boldsymbol{\theta}_{\mathrm{r}}\right) & \mathbf{L}_{\mathrm{rr}}^{\prime}
\end{array}\right]\left[\begin{array}{l}
\mathbf{i}_{\text {abcs }} \\
\mathbf{i}_{\text {abcr }}^{\prime}
\end{array}\right]
$$

And finally the matrices for the winding inductances are defined as:

$$
\mathbf{L}_{\mathrm{ss}}=\left[\begin{array}{ccc}
L_{l s}+L_{m s} & -\frac{1}{2} L_{m s} & -\frac{1}{2} L_{m s} \\
-\frac{1}{2} L_{m s} & L_{l s}+L_{m s} & -\frac{1}{2} L_{m s} \\
-\frac{1}{2} L_{m s} & -\frac{1}{2} L_{m s} & L_{l s}+L_{m s}
\end{array}\right]
$$




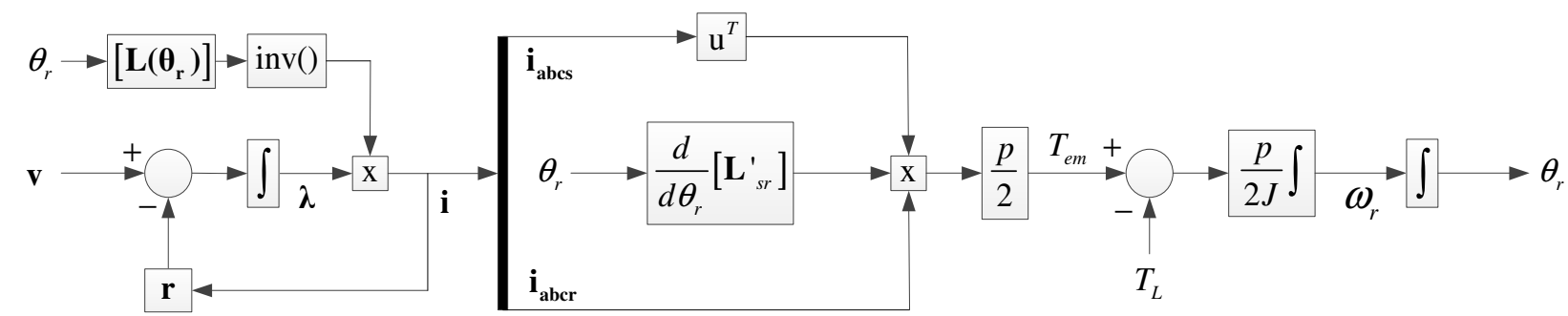

Fig. 1: Simulation of a symmetrical 3-phase induction machine in the $a b c$-reference frame shown in block diagram form

$$
\begin{gathered}
\mathbf{L}_{\mathrm{rr}}^{\prime}=\left[\begin{array}{ccc}
L_{l r}^{\prime}+L_{m r} & -\frac{1}{2} L_{m r} & -\frac{1}{2} L_{m r} \\
-\frac{1}{2} L_{m r} & L_{l r}^{\prime}+L_{m r} & -\frac{1}{2} L_{m r} \\
-\frac{1}{2} L_{m r} & -\frac{1}{2} L_{m r} & L^{\prime}{ }_{l r}+L_{m r}
\end{array}\right] \\
\mathbf{L}_{\mathrm{sr}}^{\prime}=L_{m s}\left[\begin{array}{ccc}
\cos \left(\theta_{r}\right) & \cos \left(\theta_{r}+\frac{2 \pi}{3}\right) & \cos \left(\theta_{r}-\frac{2 \pi}{3}\right) \\
\cos \left(\theta_{r}-\frac{2 \pi}{3}\right) & \cos \left(\theta_{r}\right) & \cos \left(\theta_{r}+\frac{2 \pi}{3}\right) \\
\cos \left(\theta_{r}+\frac{2 \pi}{3}\right) & \cos \left(\theta_{r}-\frac{2 \pi}{3}\right) & \cos \left(\theta_{r}\right)
\end{array}\right] \text { (6) } \\
\mathbf{L}_{\mathrm{rs}}^{\prime}=L_{m s}\left[\begin{array}{ccc}
\cos \left(\theta_{r}\right) & \cos \left(\theta_{r}-\frac{2 \pi}{3}\right) & \cos \left(\theta_{r}+\frac{2 \pi}{3}\right) \\
\cos \left(\theta_{r}+\frac{2 \pi}{3}\right) & \cos \left(\theta_{r}\right) & \cos \left(\theta_{r}-\frac{2 \pi}{3}\right) \\
\cos \left(\theta_{r}-\frac{2 \pi}{3}\right) & \cos \left(\theta_{r}+\frac{2 \pi}{3}\right) & \cos \left(\theta_{r}\right)
\end{array}\right] \text { (7) }
\end{gathered}
$$

where $\theta_{r}$ is the rotor angle, $L_{l s}$ is the stator winding leakage inductance per phase, $L^{\prime}{ }_{l r}$ is the rotor winding leakage inductance per phase, $L_{m s}$ is the self-inductance of the stator winding, $L_{m r}$ is the self-inductance of the rotor winding and the superscript ' denotes variables and parameters referred to the stator.

The electromagnetic torque $T_{e m}$ is given by the following relation in Newton· meters $(\mathrm{N} \cdot \mathrm{m})$ :

$$
T_{e m}=\left(\frac{p}{2}\right)\left(\mathbf{i}_{\text {abcs }}\right)^{T} \frac{d}{d \theta_{r}}\left[\mathbf{L}_{\text {sr }}^{\prime}\right] \mathbf{i}_{\text {abcr }}^{\prime}
$$

and the relation between the rotor speed and the torque is

$$
\frac{d \omega_{r}}{d t}=\left(\frac{p}{2}\right) \frac{T_{e m}-T_{L}}{J}
$$

Where $J$ is the inertia of the rotor in kilogram.meter ${ }^{2}$ $\left(\mathrm{kg} \cdot \mathrm{m}^{2}\right), T_{L}$ is the load torque and $p$ number of poles.

The rotor speed is the derivative of the rotor angle with respect to time so we can rewrite the equation above in the following manner:

$$
\frac{d^{2} \theta_{r}}{d t^{2}}=\frac{T_{e m}-T_{L}}{J}
$$

The terminals of the stator windings of are connected to an external voltage source and the terminals of the rotor windings are either short circuited (squirrel-cage) or can also be connected to an external voltage source. In any case, the voltage vector is known so it makes sense to rearrange (1) and combine it with (3) in the following manner:

$$
\mathbf{i}_{\mathbf{n}}=\mathbf{L}_{\mathbf{n}}^{-1}\left(\boldsymbol{\theta}_{\mathbf{r}}\right) \times \int\left(\mathbf{v}_{\mathbf{n}}-\mathbf{r} \times \mathbf{i}_{\mathbf{n}-1}\right) d t
$$

where the subscript ' $n$ ' denotes values from the current timestep and ' $n-1$ ' from the previous. In order to simulate the symmetrical three-phase induction machine the initial values for the current vector $\mathbf{i}$ and the rotor angle $\theta_{r}$ must be calculated i.e. set to zero in case of a free acceleration. The simulation process is as follows. The difference between the voltage vector and the voltage drop due to the resistance is integrated and multiplied with the inverted induction matrix. It must be mentioned here that the induction matrix $\mathbf{L}$ is a function of the rotor angle $\theta_{r}$ and it must be inverted each timestep. The obtained values for the current vector $\mathbf{i}$ are fed into (8) so the electromagnetic torque $T_{e m}$ could be calculated. Finally, the rotor angle $\theta_{r}$ is calculated according to (10). The entire simulation process of a symmetrical induction machine in the $a b c$-reference frame is depicted in Fig. 1.

\section{Proposed model}

The inductance matrix $\mathbf{L}$ from (3) is a nonsingular square matrix:

$$
\mathbf{L}\left(\boldsymbol{\theta}_{\mathrm{r}}\right)=\left[\begin{array}{cc}
\mathbf{L}_{\mathrm{ss}} & \mathbf{L}_{\mathrm{sr}}^{\prime}\left(\boldsymbol{\theta}_{\mathrm{r}}\right) \\
\mathbf{L}_{\mathrm{rs}}^{\prime}\left(\boldsymbol{\theta}_{\mathrm{r}}\right) & \mathbf{L}_{\mathrm{rr}}^{\prime}
\end{array}\right]
$$

Let its inverse matrix $\mathbf{L}_{\text {block }}^{-1}$ be defined as:

$$
\mathbf{L}_{\text {block }}^{-1}=\left[\begin{array}{cc}
\mathbf{E} & \mathbf{F} \\
\mathbf{G} & \mathbf{H}
\end{array}\right]
$$

In order to differentiate the inverted matrix obtained using matrix partitioning the subscript 'block' is used. Since $\mathbf{L}_{\mathrm{ss}}, \mathbf{L}_{\mathrm{sr}}^{\prime}, \mathbf{L}_{\mathrm{rs}}^{\prime}$ and $\mathbf{L}_{\mathrm{rr}}^{\prime}$ have the sizes $3 \times 3$, than the sizes of $\mathbf{E}, \mathbf{F}, \mathbf{G}$ and $\mathbf{H}$ must also be $3 \times 3$ [4]. Having a closer look of (4), (5), (6) and (7) it can be concluded that $\mathbf{L}_{s s}$ and $\mathbf{L}_{s r}^{\prime}$ are symmetrical matrices ( $\mathbf{L}_{\mathrm{ss}}=\left(\mathbf{L}_{\mathrm{ss}}\right)^{\mathrm{T}}$ and $\left.\mathbf{L}_{\mathrm{sr}}^{\prime}=\left(\mathbf{L}_{\mathrm{sr}}^{\prime}\right)^{\mathbf{T}}\right)$ and that $\mathbf{L}_{\mathrm{rs}}^{\prime}=\left(\mathbf{L}_{\mathrm{sr}}^{\prime}\right)^{\mathbf{T}}$. Assuming that $\mathbf{L}_{\mathrm{ss}}$ and $\mathbf{L}_{\mathrm{rr}}^{\prime}$ are nonsingular and

$$
\begin{aligned}
& S=L_{r r}^{\prime}-\left(L_{s r}^{\prime}\right)^{T}\left(L_{s s}\right)^{-1} L_{s r}^{\prime} \\
& T=L_{s s}-L_{s r}^{\prime}\left(L_{r r}^{\prime}\right)^{-1}\left(L_{s r}^{\prime}\right)^{T}
\end{aligned}
$$




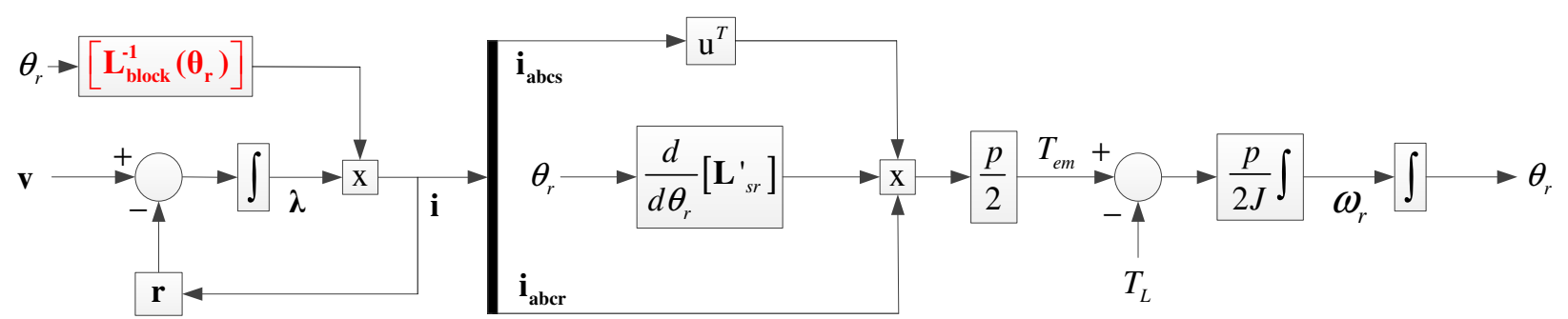

Fig. 2: The modified simulation process of a symmetrical 3-phase induction machine in the $a b c$-reference frame

Then according to [5], if the indicated inverses exist, the following expressions are equal to $\mathbf{L}_{\mathbf{b l o c k}}^{-1}$ :

$$
\begin{aligned}
& {\left[\begin{array}{cc}
\mathbf{L}_{\mathrm{ss}}^{-1}+\mathbf{L}_{\mathrm{ss}}^{-1} \mathbf{L}_{\mathrm{sr}}^{\prime} \mathbf{S}^{-1}\left(\mathbf{L}_{\mathrm{sr}}^{\prime}\right)^{\mathrm{T}} \mathbf{L}_{\mathrm{ss}}^{-1} & -\mathbf{L}_{\mathrm{ss}}^{-1} \mathbf{L}_{\mathrm{sr}}^{\prime} \mathbf{S}^{-1} \\
\mathbf{S}^{-1}\left(\mathbf{L}_{\mathrm{sr}}^{\prime}\right)^{\mathrm{T}} \mathbf{L}_{\mathrm{ss}}^{-1} & \mathbf{S}^{-1}
\end{array}\right]} \\
& {\left[\begin{array}{cc}
\mathbf{T}^{-1} & -\mathbf{T}^{-1} \mathbf{L}_{\mathrm{sr}}^{\prime}\left(\mathbf{L}_{\mathrm{rr}}^{\prime}\right)^{-1} \\
-\left(\mathbf{L}_{\mathrm{rr}}^{\prime}\right)^{-1} \mathbf{L}_{\mathrm{rs}}^{\prime} \mathbf{T}^{-1} & \left(\mathbf{L}_{\mathrm{rr}}^{\prime}\right)^{-1}+\left(\mathbf{L}_{\mathrm{rr}}^{\prime}\right)^{-1} \mathbf{L}_{\mathrm{rs}}^{\prime} \mathbf{T}^{-1} \mathbf{L}_{\mathrm{sr}}^{\prime}\left(\mathbf{L}_{\mathrm{rr}}^{\prime}\right)^{-1}
\end{array}\right]}
\end{aligned}
$$

In the equations above the matrices of the mutual inductances $\mathbf{L}_{\text {rs }}^{\prime}$ and $\mathbf{L}_{\text {sr }}^{\prime}$ are functions of the rotor angle $\theta_{r}$, however, if (14) and (15) are closely inspected, it can be seen that their values are constant and not dependent on the rotor angle. This is due to the fact that all submatrices of $\mathbf{L}$ share the same eigensystem i.e. they commute [6], so $\mathbf{S}$ and $\mathbf{T}$ could be rearranged in the following manner:

$$
\begin{aligned}
& S=L_{r r}^{\prime}-\left(L_{s r}^{\prime}\right)^{T} L_{s r}^{\prime}\left(L_{s s}\right)^{-1} \\
& T=L_{s s}-L_{s r}^{\prime}\left(L_{s r}^{\prime}\right)^{T}\left(L_{r r}^{\prime}\right)^{-1}
\end{aligned}
$$

Due to the nature of the mutual induction matrices it could easily be proven that the product $\mathbf{L}_{\text {sr }}^{\prime}\left(\mathbf{L}_{\text {sr }}^{\prime}\right)^{\mathbf{T}}$ is always constant and therefore the values of $\mathbf{S}$ and $\mathbf{T}$ are also constant.

Since both (16) and (17) are equal to $\mathbf{L}^{-1}$ we can combine them in the following manner:

$$
\mathbf{L}_{\text {block }}^{-1}\left(\theta_{r}\right)=\left[\begin{array}{cc}
\mathbf{T}^{-1} & -\mathbf{L}_{\mathrm{ss}}^{-1} \mathbf{L}_{\mathrm{sr}}^{\prime}\left(\boldsymbol{\theta}_{\mathrm{r}}\right) \mathbf{S}^{-1} \\
-\mathbf{S}^{-1}\left(\mathbf{L}_{\mathrm{sr}}^{\prime}\left(\boldsymbol{\theta}_{\mathrm{r}}\right)\right)^{\mathrm{T}} \mathbf{L}_{\mathrm{ss}}^{-1} & \mathbf{S}^{-1}
\end{array}\right]
$$

Now, it is easily seen that the submatrices in the main diagonal are always constant and that only the submatrices in the secondary diagonal should be calculated each timestep. In order to reduce the computation effort even further one could take advantage of the fact that the selfinductance matrices $\mathbf{L}_{\mathrm{ss}}$ and $\mathbf{L}_{\text {rr }}^{\prime}$ are symmetric i.e. equal to their transpose. That yields in the fact that the upper right part of $\mathbf{L}_{\text {block }}^{-1}$ is equal to the lower left part. Finally, the expression for the inverted induction matrix looks as follows:

$$
\begin{aligned}
& \mathbf{L}_{\text {block }}^{-1}\left(\boldsymbol{\theta}_{\mathbf{r}}\right)=\left[\begin{array}{cc}
\mathbf{T}^{-1} & -\mathbf{U L}_{\mathrm{sr}}^{\prime}\left(\boldsymbol{\theta}_{\mathbf{r}}\right) \\
\left(-\mathbf{U L}_{\mathrm{sr}}^{\prime}\left(\boldsymbol{\theta}_{\mathbf{r}}\right)\right)^{\mathbf{T}} & \mathbf{S}^{-1}
\end{array}\right] \\
& \mathbf{U}=\mathbf{L}_{\mathrm{ss}}^{-1} \mathbf{S}^{-1}=\text { const }
\end{aligned}
$$

In this manner, the inversion of the $6 \times 6$ matrix reduces to multiplication of two $3 \times 3$ matrices and transposing their product which considerably lowers the computation effort. The modified simulation process is depicted in Fig. 2 where the changes with regard to Fig. 1 are marked in red.

The difference is, of course, the fact that no longer matrix inversion per se is needed. Instead, the inverted induction matrix is now only a function of the rotor angle $\theta_{r}$.

\section{Model Verification}

The mathematical model of the symmetrical 3-phase induction machine, as described in the previous chapter and depicted in Fig. 2, was implemented in MATLAB as a script, but this can also be done in practically any programming language. In order to verify its behavior it has been compared against the model depicted in Fig. 1 which has also been implemented as a MATLAB script. In order to compare the two models, the variables of the both induction machine models during free (no-load) acceleration were observed. The parameters of the machine are from [7] and given in Table I.

Table I Induction Motor Parameters

\begin{tabular}{|l|l|}
\hline Rated power $S_{n}$ & $1.5 \mathrm{MW}$ \\
\hline Rated line voltage $U_{n}$ & $690 \mathrm{~V}(\mathrm{~L}-\mathrm{L}, \mathrm{rms})$ \\
\hline Rated frequency $f_{s}$ & $50 \mathrm{~Hz}$ \\
\hline Phases & 3 \\
\hline Number of poles $p$ & 6 \\
\hline Moment of Inertia $J$ & $70 \mathrm{~kg} \cdot \mathrm{m}^{2}$ \\
\hline Stator resistance $R_{s}$ & $0.002 \Omega$ \\
\hline Rotor resistance $R_{r}$ & $0.0015 \Omega$ \\
\hline Stator inductance $L_{l s}$ & $1.5915 \mathrm{e}-04 \mathrm{H}$ \\
\hline Rotor inductance $L_{l r}$ & $1.4961 \mathrm{e}-04 \mathrm{H}$ \\
\hline Mutual inductance $L_{m}$ & $0.0018 \mathrm{H}$ \\
\hline
\end{tabular}

Fig. 3 shows the free acceleration characteristic of the induction machine from a stationary state to its steady state. From the figure the transient behavior of the stator current in the phase $A$, the transient behavior of the rotor current in the phase $A$, the electromagnetic torque, the rotor speed and the torque-speed characteristic of the induction can be observed.

After 9 seconds of the simulation time the steady-state operation is reached. In steady-state operation the amplitude of the stator current reaches 619.2 A, the amplitude of the rotor current is almost equal to $0 \mathrm{~A}$ and 

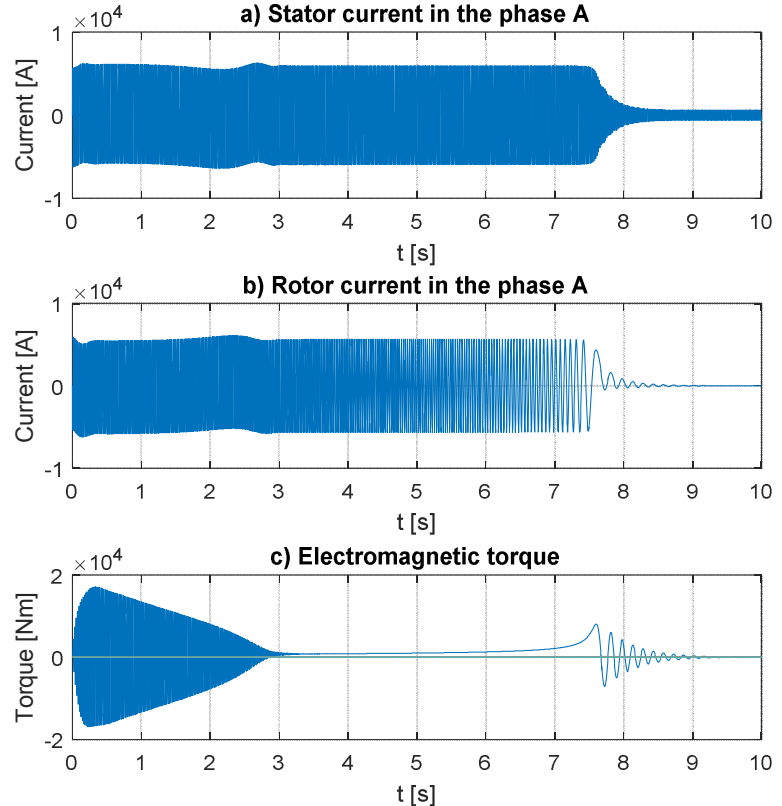

d) Rotor speed
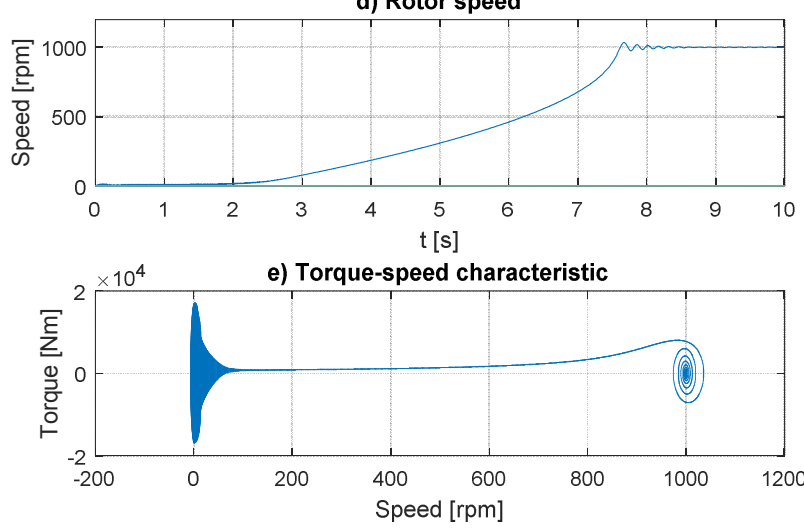

Fig. 3 The free acceleration characteristic

since the motor is working without load the slip is close to zero i.e. the frequency of the rotor is practically $0 \mathrm{~Hz}$.

This is due to the fact that $f_{r}=s \cdot f_{s}$, where $f_{r}$ and $f_{s}$ are the rotor and stator frequencies respectively and $s$ is the rotor slip.

The rotor speed of the unloaded induction machine is given by $n=120 \cdot f_{s} / p$ and is equal to $1000 \mathrm{rpm}$, which corresponds to the simulation results shown in Fig. 3-d.

The free acceleration characteristic shown in Fig. 3 is absolutely identical for the both implementations of the induction machine i.e. the implementation which inverts the induction matrix of the machine and the implementation according to the proposed model from Chapter 3 in this paper.

The only difference between the two implementations can be seen in the computation effort needed for their simulation. The results are presented in the following chapter.

\section{Simulation performance}

As already mentioned the computational effort needed for the proposed model is considerably lower than the conventional method which relies on matrix inversion. In order to compare the performances of both models their execution times were measured. The whole performance

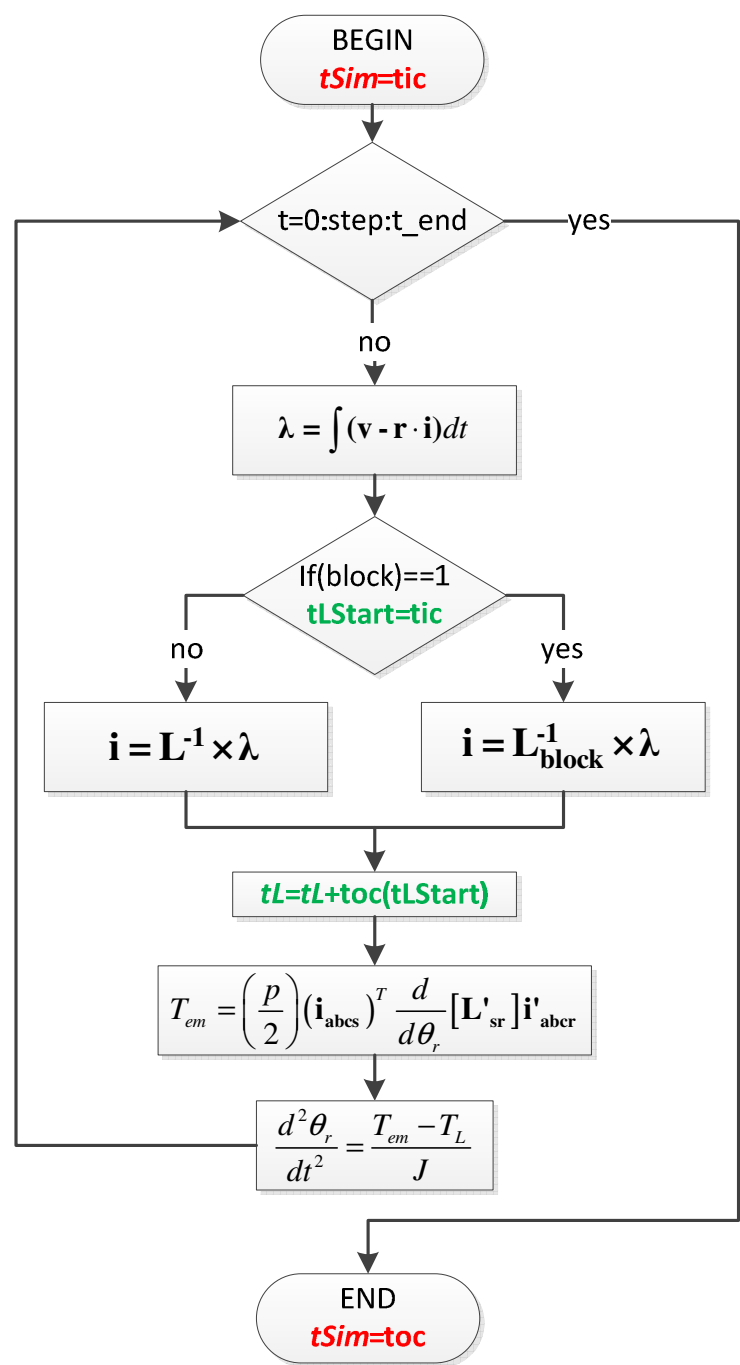

Fig. 4: The performance measurement process

measurement process is depicted in the flowchart shown in Fig. 4. The performance is measured by the two timers which are implemented in the simulation. In the simulation, if the variable block is set to 1 , then the proposed method for matrix inversion is used. The first timer tSim, which is marked in red in the figure, is measuring the whole simulation time from the beginning to the end. The second timer $t L$, which is marked in green in the figure, measures only the time needed for the calculation of the inverted induction matrix.

The whole process shown in Fig. 4 is also implemented as a MATLAB script and the timers $t$ Sim and $t L$ are using the built functions for time measurement tic and toc. The keyword tic starts the timer [8], whereas the keyword toc returns the elapsed time [9].

The results of the performance measurement are summarized in Table II.

Table II Execution times of $t \operatorname{Sim}$ and $t L$

\begin{tabular}{|l|c|c|c|}
\hline & block=0 & block=1 & Difference [\%] \\
\hline tSim [s] & 12.1013 & 9.3191 & 22.9907 \\
\hline tL [s] & 3.9654 & 2.4048 & 39.3557 \\
\hline
\end{tabular}

From the table it can be seen that the inversion of the inductance matrix using the proposed model $($ block $=1)$ is almost $40 \%$ faster than the inversion using the 
conventional method (block=0). For the conventional method the MATLAB built-in function $\operatorname{inv}()$ was used, which forms the explicit inverse of a matrix. The same test was also performed using the mldivide() function, which produces solution using Gaussian elimination [10], but there were no significant performance improvements in comparison to the $\operatorname{inv}()$ function.

The total simulation time, measured by $t \operatorname{Sim}$, is not as decisive as $t L$ since it also depends on the total extent of the simulation. However, for the current case shown on Fig. 4, an improvement of almost $23 \%$ can be seen.

\section{Conclusion}

In this paper an alternative mathematical model in the $a b c$ reference frame for the simulation of a symmetrical induction machine was developed. This mathematical model takes advantage of the matrix inversion of partitioned matrices. The developed model was implemented in the MATLAB and compared against the conventional one. The comparison, which was done by observing the variables of the both models during free (noload) acceleration, verified that there was no difference between the conventional and the proposed method.

The performance analysis revealed that there is a significant reduction in the execution times in favor of the proposed model.

The developed model is only valid for symmetrical induction machines.

The model could be used as a faster alternative to the conventional $a b c$-reference frame method. Another possible application could be the faster and more intuitive simulation of the slot harmonics in the induction machines.

\section{References}

[1] R. H. Park, "Two-reaction theory of synchronous machines generalized method of analysis-part I," Trans. Am. Inst. Electr. Eng., vol. 48, no. 3, pp. 716727, 1929.

[2] H. R. Fudeh and M. Ieee, "Modeling and Analysis of Induction Machines Part III: Three-Phase Cage Rotor Induction Machines," Power Appar. Syst. IEEE Trans., vol. Volume:PAS, no. 8, 1983.

[3] P. Krause, O. Wasynczuk, S. Sudhoff, and S. Pekarek, Analysis of electric machinery and drive systems 3e, 3rd ed. Wiley-IEEE Press, 2013.

[4] T. T. Lu and S. H. Shiou, "Inverses of $2 \times 2$ block matrices," Comput. Math. with Appl., vol. 43, no. 12, pp. 119-129, 2002.

[5] F. Zhang, The Schur complement and its applications, vol. 4. Springer US, 2005.

[6] M. P. Drazin, "Some generalizations of matrix commutativity," Proc. London Math. Soc., vol. 3, no. 1,1951 .

[7] N. Mohan, Advanced Electric Drives: Analysis, Control and Modeling Using Simulink, 1 edition. Wiley, 2014.

[8] "Start stopwatch timer - MATLAB tic - MathWorks Deutschland." [Online]. Available: http://de.mathworks.com/help/matlab/ref/tic.html. [Accessed: 13-Oct-2015].

[9] "Read elapsed time from stopwatch - MATLAB toc - MathWorks Deutschland." [Online]. Available: http://de.mathworks.com/help/matlab/ref/toc.html. [Accessed: 13-Oct-2015].

[10] "Matrix inverse - MATLAB inv - MathWorks Deutschland." [Online]. Available: http://de.mathworks.com/help/matlab/ref/inv.html. [Accessed: 14-Oct-2015]. 